\title{
Patients with Cirrhosis of Liver Operated for Non-transplant Surgery: A Retrospective Analysis
}

\author{
Kishore Kumar Kuduva Subramanian ${ }^{1}$, Manish Tandon* ${ }^{1}$, Chandra Kant Pandey ${ }^{1}$ \\ and Priyanka Jain ${ }^{2}$ \\ ${ }^{1}$ Department of Anaesthesiology, Institute of Liver and Biliary Sciences, New Delhi, India; ${ }^{2}$ Department of Research, Institute of \\ Liver \& Biliary Sciences (ILBS), New Delhi, India
}

\section{Abstract}

Background and Aims: Patients with cirrhosis of the liver have high mortality after surgery. We investigated the mortality in patients with cirrhosis of the liver who underwent surgery other than liver transplant and applied the Mayo clinic model to predict mortality and compare with the observed mortality. We also studied the association of the observed mortality with the Child-Turcotte-Pugh (CTP) class and the model for end-stage liver disease (MELD) and model for end-stage liver disease-sodium (MELD-Na) scores. Methods: The electronic records database of our hospital was accessed to analyze the data of 133 cirrhotic patients who underwent various surgeries under general anesthesia from October 2009 to June 2017. The Mayo risk score was applied to each and used to calculate predicted mortality; the MELD and MELD-Na scores were also calculated. Telephonic interview was performed with the patients and or their relative to ascertain survival or time of death after surgery, when the information was not available from the hospital records. Results: The all-cause observed mortality rates at postoperative days 30 and 90 and at 1 year were $12 \%, 20.3 \%$ and $26.3 \%$ respectively. The area under the receiver operating characteristic curve values for the Mayo model as a predictor of 30-day, 90-day and 1-year mortality were $0.836,0.828$ and 0.744 respectively. Good correlation was seen for observed mortality with CTP class and with MELD and MELD-Na scores. Conclusions: The Mayo model for predicting postoperative mortality in patients with cirrhosis of the liver demonstrated good correlation in this study. The strength of prediction of mortality by Mayo risk score calculation was similar at postoperative days 30 and 90 but decreased at 1-year after the surgery. Good correlation was seen for the observed mortality with MELD, MELD-Na and CTP scores.

Citation of this article: Subramanian KKK, Tandon M, Pandey CK, Jain P. Patients with cirrhosis of liver operated for non-transplant surgery: A retrospective analysis. J Clin Transl Hepatol 2019;7(1):9-14. doi: 10.14218/JCTH.2018.00043.

Keywords: Cirrhosis of the liver; Mayo risk model; CTP score; MELD score. Abbreviations: AUROC, area under the receiver operating characteristic curve; ASA, American Society of Anesthesiologists; CI, confidence interval; CTP, ChildTurcotte-Pugh; MELD, model for end-stage liver disease; MELD-Na, model for endstage liver disease-sodium; OR, odds ratio.

Received: 13 July 2018; Revised: 15 November 2018; Accepted: 9 January 2019 *Correspondence to: Manish Tandon, Department of Anaesthesiology, Institute of Liver and Biliary Sciences, D-1, Vasant kunj, New Delhi, India. Tel: +919871437478, Fax: +91-1146300010, E-mail: manishtandon25@rediffmail.com

\section{Introduction}

Chronic liver disease is a multi-system disorder which leads to decreased quality of life and life expectancy. Patients with cirrhosis are also at increased risk of morbidity and mortality after surgery. ${ }^{1-3}$ Postoperative mortality after non-transplant surgery is $11-25 \%$ for cirrhotic patients, compared to $1.1 \%$ for patients without cirrhosis of the liver. ${ }^{4}$ Patient selection and prognostication is therefore crucial. Several risk scoring systems, including the American Society of Anesthesiologists (ASA) physical status classification, Child-Turcotte-Pugh (CTP) classification and model for end-stage liver disease (MELD) scoring, are used to prognosticate outcomes and risk associated with surgery. However, unlike any of those listed above, the Mayo postoperative mortality risk prediction model was designed specifically to predict mortality after non-transplant surgery for patients with cirrhosis of the liver.

We performed a retrospective analysis of the patients with cirrhosis of the liver who had been operated upon with surgeries other than liver transplant surgery at our hospital. Their expected mortality was calculated using the Mayo risk score, on the basis of each patient's preoperative investigative findings, and assessed for correlation with expected mortality in comparison to the observed mortality over a period of 1 year after surgery. We also looked for correlation of the observed mortality with the MELD and model for endstage liver disease-sodium (MELD-Na) scores and with the CTP score.

\section{Methods}

This study was approved by the Institutional Ethics Committee (Vide letter IEC/2017/49/MA11, dated 6/4/2017). The hospital's electronic information system and scanned copies of patient physical records were accessed. We identified 240 patients with cirrhosis of the liver who were operated upon for non-transplant surgery under general anesthesia between the dates of October 2009 and June 2017. Among all the patients identified by these criteria, 90 could not be traced for telephonic follow-up and data was incomplete for another 17. Complete data was available for 133 cirrhotic patients who had undergone various gastro-intestinal, hepato-pancreatico-biliary and urological surgeries, excluding liver transplant surgery. All included patients had cirrhosis documented by (i) histological findings, (ii) clinical findings, and/ or (iii) radiological imaging findings consistent with cirrhosis of the liver. 
Table 1. Demographic profile of the study population

\begin{tabular}{ll}
\hline Characteristic & $\begin{array}{l}\text { Number of } \\
\text { patients }\end{array}$ \\
\hline Total number of patients, $n$ & 133 \\
Mean age in years & $51 \pm 11.6$ \\
Sex, M/F & $112 / 21$ \\
MELD score as mean \pm SD (Survivor & $18 \pm 8.5$ \\
vs. Non-Survivor at 1 year) & $(15.4$ vs. 23.5) \\
MELD-Na score as mean \pm SD & $20 \pm 8.5$ \\
(Survivor vs. Non-Survivor at 1 year) & $(18.4 \mathrm{vs.} \mathrm{23.9)}$ \\
CTP class, $n$ (\%) for A & $12(9 \%)$ \\
CTP class, $n$ (\%) for B & $83(62 \%)$ \\
CTP class, $n$ (\%) for C & $38(29 \%)$ \\
CTP score as mean \pm SD (Survivor & $8.6 \pm 2.6$ \\
vs. Non-Survivor) & $(8.1$ vs. 10.0$)$ \\
ASA status, $n$ (\%) for III & $114(86 \%)$ \\
ASA status, $n$ (\%) for IV & $19(14 \%)$ \\
Emergency surgery, $n$ (\%) & $37(28 \%)$ \\
Etiology of cirrhosis, $n$ (\%) & \\
Alcoholic & $46(35 \%)$ \\
Viral & $40(30 \%)$ \\
Non-alcoholic steatohepatitis & $21(15 \%)$ \\
Cryptogenic & $15(11 \%)$ \\
Others* & $11(9 \%)$ \\
Observed mortality at 30 days, $n(\%)$ & $16(12 \%)$ \\
Observed mortality at 90 days, $n(\%)$ & $27(20.3 \%)$ \\
Observed mortality at 1 year, $n(\%)$ & $35(20.3 \%)$ \\
\hline
\end{tabular}

*others: Non-cirrhotic portal fibrosis - 3, autoimmune hepatitis - 2, hepatic venous outflow tract obstruction -2 , primary biliary cholangitis -1 , primary sclerosing cholangitis -1 , extrahepatic portal venous obstruction -1 , chronic cholestatic liver disease -1 .

Elective as well as emergency surgeries were included in the study. Each patient was categorized by an anesthesiologist before the surgery into one of the five ASA classes. ${ }^{5}$ The CTP score, MELD and MELD-Na scores were calculated. ${ }^{6-9}$ Lengths of hospital and Intensive Care Unit stays, type of surgery, date of surgery, and date of death of the patient (whenever applicable) were obtained. Patients discharged to home were contacted by telephone to ascertain their outcome. Postoperative mortality risk was calculated on the website provided by the Mayo clinic (http://www.mayoclinic. org/meld/mayomodel9.html). ${ }^{10}$ Follow-up was conducted for up to 1 year or until the time of death within the 1 year following the surgery. Patients diagnosed with cirrhosis after the surgery and patients with acute liver failure and/or acuteon-chronic liver disease were not included. Patients who underwent liver transplant surgery before or during the study period were also excluded from the study.

\section{Statistical analysis}

Categorical variables were presented as proportions, while continuous variables were presented either as mean with standard deviation or median with range. Comparison of two continuous variables was done by independent $t$-test or Mann-Whitney test for parametric or nonparametric data respectively, and categorical variables by Fisher's exact test or Pearson's chi-square test. Pearson's or Spearman's correlation coefficient was used for measuring any association between variables. Univariate regression analysis was used for calculating risk for disease severity score. All statistical analyses were two-tailed and used a significance level $(p)$ of $<0.05$, and were carried out by the SPSS statistical software for Windows (version 22; IBM Corp., Armonk, NY, USA).

\section{Results}

A total of 133 patients diagnosed with cirrhosis of the liver were included in this study. Males formed the majority of the study population, accounting for $84 \%(n=112)$, and the mean age was 51 years. The average MELD score was 17 , MELD-Na score was 20, CTP score was 9. Out of the 133 patients, 12, 83 and 38 were grouped into CTP classes A, B and $C$ respectively. One hundred and fourteen patients ( $86 \%)$ were classified as ASA class III, and the rest (14\%) as ASA class IV. Thirty-seven patients (28\%) were operated upon for emergency surgical intervention (Table 1).

No patient was hypertensive or had diabetes mellitus at the time of the diagnosis of cirrhosis of the liver. Unconfirmed history of hypertension was present in 17 patients, but at the time of diagnosis of cirrhosis of the liver no patient was found to be hypertensive. Renal dysfunction or a history of renal dysfunction secondary to cirrhosis of the liver was present in 108 of 133 patients. Alcoholic liver disease (35\%) was closely

Table 2. Area under the receiver operating characteristic curves for the observed mortality and different scores

\begin{tabular}{|c|c|c|c|c|c|}
\hline & Area under the curve & Cutoff value & Sensitivity & Specificity & $p$ \\
\hline Mayo 30 days & 0.84 & 29.5 & 73.3 & 73.0 & $<0.001$ \\
\hline Mayo 90 days & 0.83 & 41.5 & 73.0 & 75.0 & $<0.001$ \\
\hline Mayo 1 year & 0.75 & 41.0 & 67.6 & 69.7 & $<0.001$ \\
\hline MELD 30 days & 0.83 & 19.5 & 75.0 & 72.6 & $<0.001$ \\
\hline MELD 90 days & 0.83 & 18.5 & 74.1 & 73.6 & $<0.001$ \\
\hline MELD 1 year & 0.76 & 16.5 & 68.6 & 63.0 & $<0.001$ \\
\hline MELD-Na 30 days & 0.77 & 24.5 & 75.0 & 74.5 & 0.001 \\
\hline MELD-Na 90 days & 0.74 & 21.5 & 66.7 & 67.0 & 0.001 \\
\hline MELD-Na 1 year & 0.67 & 20.5 & 60.0 & 60.2 & 0.003 \\
\hline
\end{tabular}




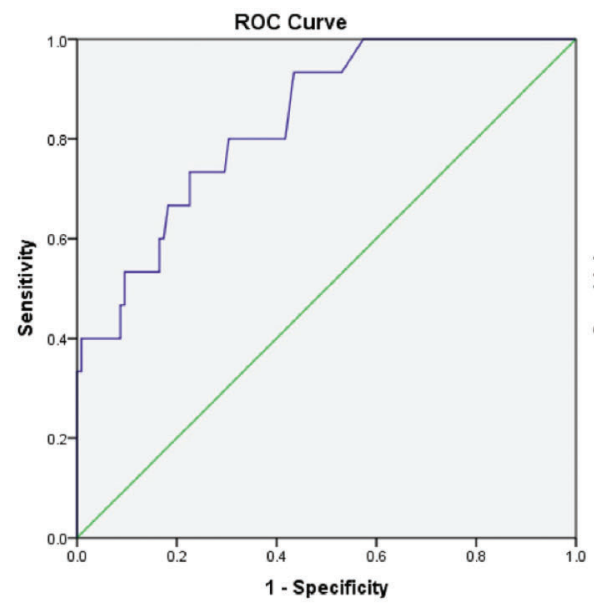

Diagonal segments are produced by ties.

Mortality at 30 days

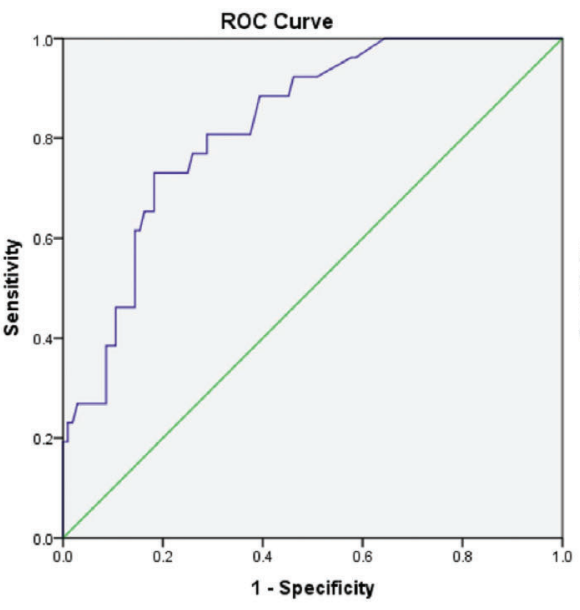

Diagonal segments are produced by ties.

Mortality at 90 days

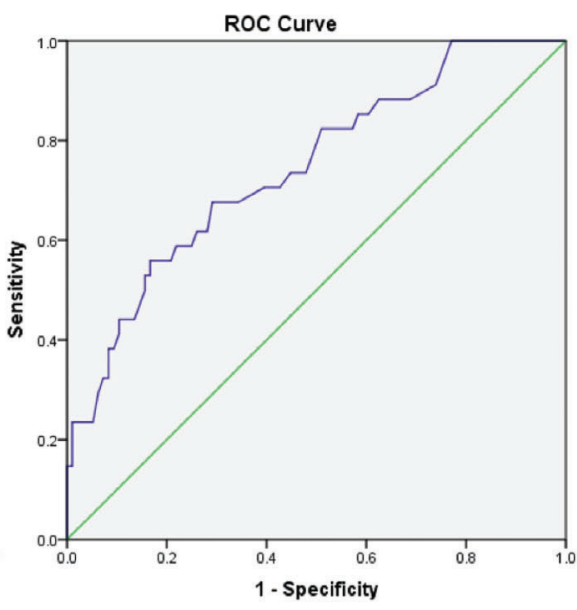

Diagonal segments are produced by ties.

Mortality at 1 year

Fig. 1. Receiver operating characteristic curves for MAYO score predicted mortality.

followed by viral etiology (30\%) as the cause for the cirrhosis of the liver. The overall 30-day, 90-day and 1-year observed mortality rates were $12 \%, 20.3 \%$ and $26.3 \%$ respectively.

There was a good correlation between the observed mortality and the predicted mortality, as calculated by using the Mayo risk calculator, among our study population, with the area under the receiver operating characteristic curve (commonly known as AUROC) for 30-day, 90-day and 1 -year mortality of $0.836,0.826$ and 0.744 respectively, sensitivity of $73.3 \%, 73.1 \%$ and $67.6 \%$ respectively, and specificity of $75 \%, 75 \%$ and $65.6 \%$ respectively (Table 2 ) (Fig. 1).

We plotted receiver operating characteristic curve and found a cutoff of 19.5 for MELD score and 24.5 for MELD-Na score, above which the 30-day mortality increased for every point increase in score (Table 2) (Figs. 2 and 3). We also found that for every point increase in MELD score above the cutoff value, the mortality at day 30 , day 90 and 1 year increased by $16 \%$ (odds ratio (OR): $1.16,95 \%$ confidence interval $(\mathrm{CI})$ : $1.08-1.25, p<0.001$ ), $16 \%$ (OR: 1.16 , 95\% CI: $1.09-$ $1.24, p<0.001$ ) and $12 \%$ (OR: $1.12,95 \% \mathrm{CI}: 1.07-1.18$, $p<0.001)$ respectively. Similarly, for every point increase in MELD-Na score above the cutoff value, the mortality at day 30 , day 90 and at 1 year increased by $13 \%$ (OR: $1.13,95 \%$ CI: $1.06-1.23, p<0.001$ ), $12 \%$ (OR: $1.12,95 \%$ CI: $1.06-$ $1.19, p<0.001$ ) and $8 \%$ (OR: $1.08,95 \%$ CI: $1.03-1.14, p<$ 0.001 ) respectively. Whereas, for every unit change in CTP score, the OR of mortality at day 30 was 1.86 (95\% CI: $1.38-2.50, p<0.001)$, at day 90 was 1.56 (95\% CI: $1.28-$ $1.90, p<0.001)$ and at 1 year was 1.35 (95\% CI: $1.15-1.59$, $p<0.001$ ) (Table 3).

For CTP class A, B and C, mortality at day 30 was $0 / 16$, $4 / 16$ and $12 / 16,(p<0.001)$ respectively, at day 90 was $1 /$ $27,10 / 27$ and $16 / 27$ respectively $(p<0.001)$, and at 1 year

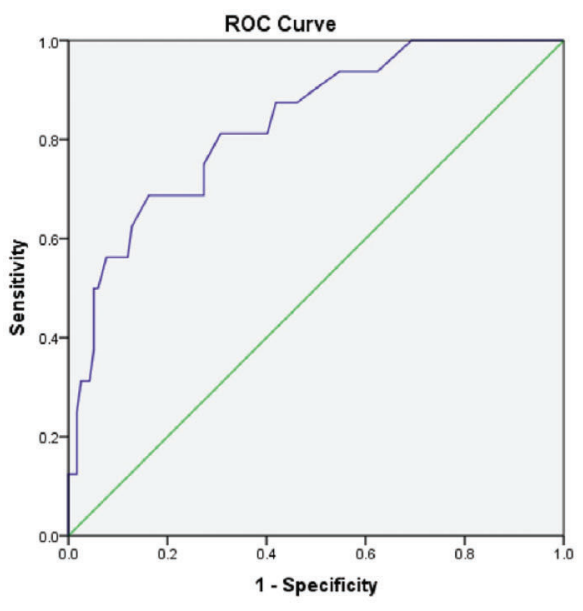

Diagonal segments are produced by ties.

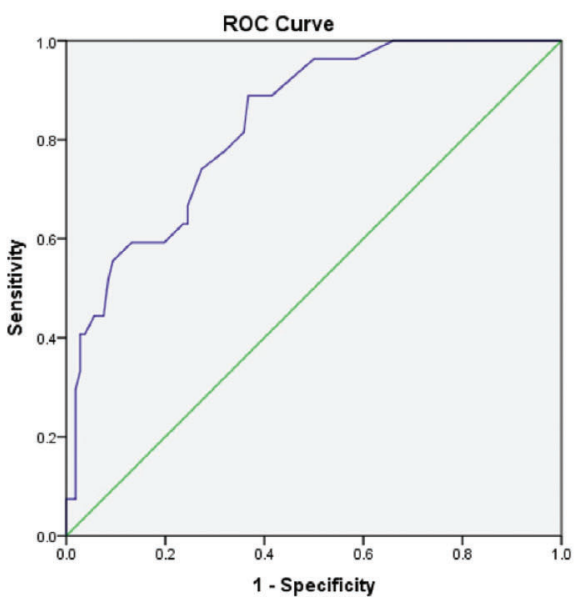

Diagonal segments are produced by ties

Mortality at 90 days

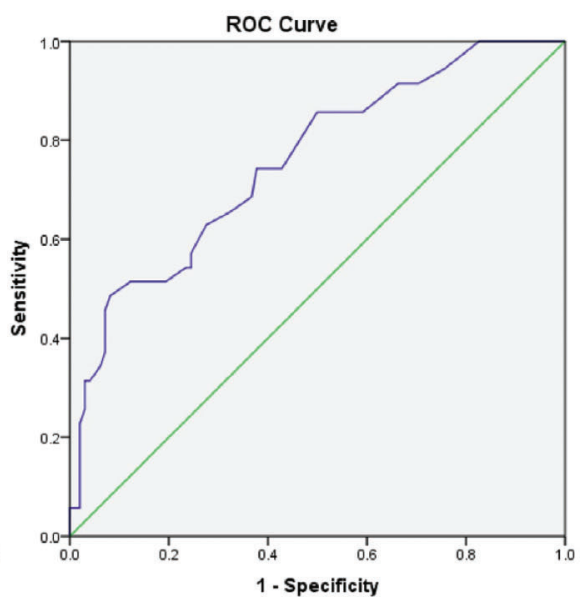

Diagonal segments are produced by ties.

Mortality at 1 year

Fig. 2. Receiver operating characteristic curves for MELD score predicted mortality. 


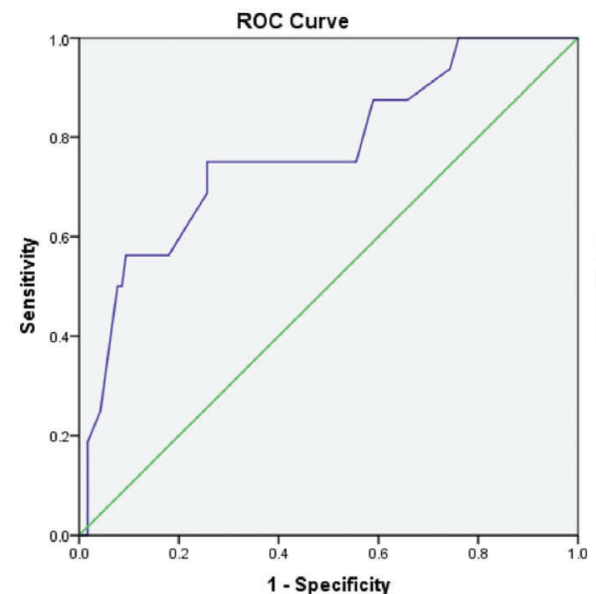

Diagonal segments are produced by ties.

Mortality at 30 days

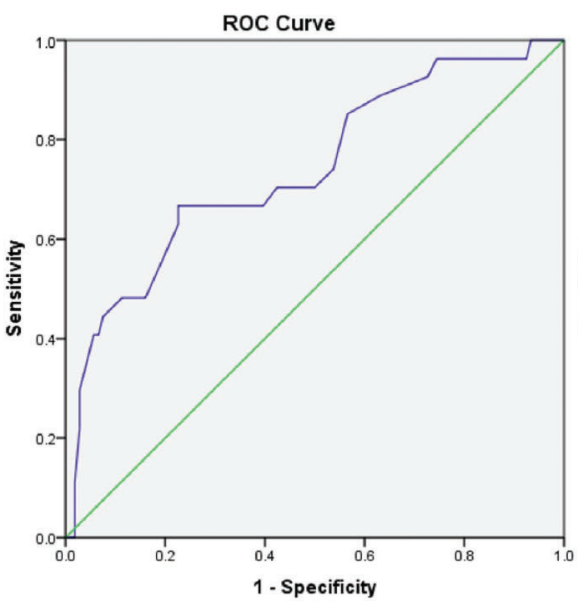

Diagonal segments are produced by ties.

Mortality at 90 days

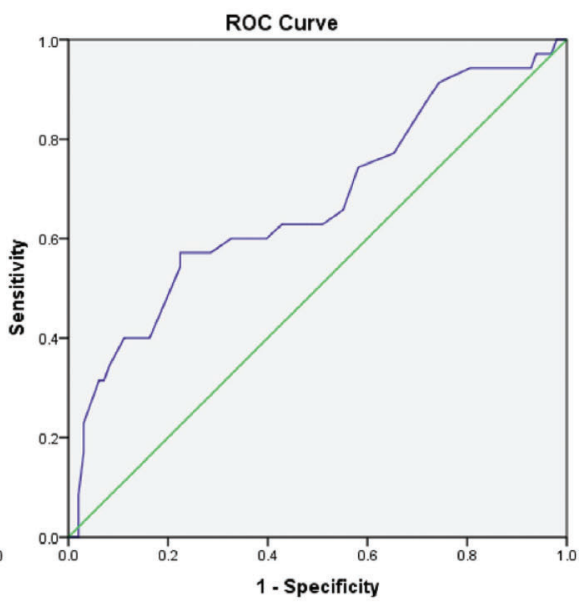

Diagonal segments are produced by ties.

Mortality at 1 year

Fig. 3. Receiver operating characteristic curves for MELD-Na score predicted mortality.

was $3 / 35,14 / 35$ and $18 / 35$ respectively $(p=0.002)$ (Table 4 ). Overall mortality in the observed period of 1 year for ASA class III and IV was 20.2 and $73.7 \%$ respectively. Emergency surgeries accounted for the significant number of mortalities in both ASA class III and IV (Table 5). Gastrointestinal and urology surgeries accounted for the majority of the surgical interventions (Table 6).

\section{Discussion}

Surgical intervention for various reasons is made in approximately $10 \%$ of patients with chronic liver disease within 2 years before their death. ${ }^{11}$ Surgery in this group of patients is associated with high mortality, of $11-25 \% .{ }^{4}$ Type of surgery, CTP score and MELD score have each been independently associated with postoperative morbidity and mortality in such patients with cirrhosis of the liver. ${ }^{12}$

The Mayo risk calculator is a tool used for prognosticating risk of mortality among patients with cirrhosis of the liver presenting for any surgery, excluding liver transplant. In this study, we analyzed 133 patients with cirrhosis of the liver who had been operated on for non-transplant surgery and found that the Mayo risk calculator was able to predict the observed mortality at day 30, day 90 and at 1 year, with AUROCs of $84 \%, 83 \%$ and $72 \%$ respectively (Table 2 ).

In a systematic review of 100 cirrhotic patients, predominantly of ethanol origin and who were undergoing abdominal surgery, the perioperative mortality rates of $10 \%, 31 \%$ and $76 \%$ were reported for the patients classified as Child's class A, B and C respectively. ${ }^{13}$ Out of 52 parameters that were assessed in a multivariate analysis, Child's classification was the best predictor of surgical mortality and morbidity. In this study, we found that in addition to CTP score, MELD and MELD-Na scores are also independent predictors of mortality at day 30 , day 90 and at 1 year (Table 3).

Teh et al. ${ }^{10}$ studied 772 patients with cirrhosis of the liver and found MELD score, age and ASA class to be statistically significant predictors of mortality. ASA class was the strongest predictor of the 7-day mortality, whereas MELD score was the best predictor of 30-day, 90-day and long-term postoperative mortality, for all types of surgery. Teh et al. ${ }^{10}$ also concluded that the relative risk of 30 - and 90 -day mortality increased by $14 \%$ with each 1 -point increase in the MELD score. Another study by Northup et al. ${ }^{15}$ concluded that $1 \%$ increase in mortality occurs for every point increase in MELD score less than 20 and that there is an additional $2 \%$ increase in mortality for every MELD point increase greater than 20. Our study also found that for every point increase in MELD score, the mortality at 30 days, 90 days and 1 year

Table 3. Univariate analysis for MELD and MELD-Na scores and CTP class

\begin{tabular}{cl}
\hline Variable & Odds ratio \\
\hline MELD score & \\
30-day mortality & $\begin{array}{l}1.16,95 \% \mathrm{CI}: 1.08-1.25, \\
p<0.001\end{array}$ \\
& $1.16,95 \% \mathrm{CI}: 1.09-1.24$, \\
& $p<0.001$ \\
90-day mortality & $1.12,95 \% \mathrm{CI}: 1.07-1.18$, \\
& $p<0.001$ \\
1-year mortality & $1.13,95 \% \mathrm{CI}: 1.06-1.23$, \\
MELD-Na score & $p<0.001$ \\
30-day mortality & $1.12,95 \% \mathrm{CI}: 1.06-1.19$, \\
& $p<0.001$ \\
90-day mortality & $1.08,95 \% \mathrm{CI}: 1.03-1.14$, \\
& $p<0.001$ \\
1-year mortality & $1.86,95 \% \mathrm{CI}: 1.38-2.50$, \\
CTP class & $p<0.001$ \\
30-day mortality & $1.56,95 \% \mathrm{CI}: 1.28-1.90$, \\
& $p<0.001$ \\
90-day mortality & $1.35,95 \% \mathrm{CI}: 1.15-1.59$, \\
& $p<0.001$ \\
\hline 1-year mortality & \\
\hline
\end{tabular}


Subramanian K.K.K. et al: Non-transplant surgery for liver cirrhosis

Table 4. Mortality and the CTP class

\begin{tabular}{llll}
\hline CTP class & 30 -day mortality & 90 -day mortality & 1 -year mortality \\
\hline A,$n=12$ & 0 & $1(3.7 \%)$ & $3(8.6 \%)$ \\
B $n=83$ & $4(25 \%)$ & $10(37 \%)$ & $14(40 \%)$ \\
C,$n=38$ & $12(75 \%)$ & $16(59.3 \%)$ & $18(51.4 \%)$ \\
Total & 16 & 27 & 35 \\
\hline
\end{tabular}

increased by $16 \%, 16 \%$ and $12 \%$ respectively and that for every 1 -point increase in MELD-Na score the mortality increased by $13 \%, 12 \%$ and $8 \%$ respectively (Table 3 ).

We also plotted the receiver operated characteristic curve and calculated the best cutoff values for predicted mortality using Mayo risk calculator. The cutoff values thus derived imply that predicted mortality, more than these cutoff values, was associated with significant risk of actual mortality in our study population. The area under the curve for mortality at 30 days was 0.84 , with significant cutoff at 29.5 having sensitivity and specificity of $73.3 \%$ and $75 \%$ respectively. For mortality at 90 days, the area under the curve was 0.83 , with cutoff at 41.5 having sensitivity and specificity of $73 \%$ and $75 \%$ respectively. Similarly, for mortality at 1 -year postsurgery, the area under the curve was 0.74 with cutoff at 38 having sensitivity and specificity of $67.6 \%$ and $65.6 \%$ respectively.

Kim et al. ${ }^{14}$ carried out a retrospective analysis to validate the Mayo postoperative risk prediction model in Korean cirrhotic patients. In their analysis, the data of 160 cirrhotic patients undergoing surgery under general anesthesia were reviewed and CTP score, MELD score, ASA class and age were found to be significant predictors of postoperative mortality in cirrhotic patients. Moreover, the Mayo model for risk prediction showed good correlation in cirrhosis of the liver among Korean patients but the model tended to overestimate mortality, especially at 1-year post-surgery. In our study also, the Mayo risk model was seen to overestimate the mortality. The mean mortality calculated by the Mayo model for our study population at postoperative day 30 , day 90 and 1 year was $26 \%, 34.5 \%$ and $41.8 \%$ respectively, which was significantly higher than the observed mortality of $12 \%, 20 \%$ and $26 \%$ respectively.

In the study by Kim et al. ${ }^{14}$ the overall 30-day, 90-day and 1 -year mortality rates were $7.5 \%, 9.4 \%$ and $10.6 \%$ respectively, as compared to $12 \%, 20.3 \%$ and $26.3 \%$ in our study. The relatively higher mortality percentage seen in our study population is probably because of the higher mean MELD score of 18 versus that of 6 in the Korean group, implying sicker patients among our study population. The implication of sicker patients in our study population is also reflected in the CTP class distribution; where $78 \%$ in the Korean study were of CTP A, only $9 \%$ of our patients were CTP A.
In a retrospective study of 140 cirrhotic patients undergoing non-transplant surgeries, the mean MELD score of survivors was compared against the mean MELD score of non-survivors (16.2 vs. 24.8). ${ }^{15} \mathrm{~A}$ similar difference was seen in our study, wherein the mean MELD score of survivors at 1 year after surgery was 15.4 , while it was 23.5 for our non-survivors. In our study, the patients who did not survive had a mean MELD-Na score of 23.9 and a mean CTP of 10 (Table 1 )

The ASA classification incorporates measures of cardiopulmonary function that are critical for determination of survival in patients with cirrhosis of the liver. ${ }^{16}$ ASA score is an independent risk factor for early as well as for late (i.e. after 1 year) mortality. ${ }^{9-13}$ In this study, we observed a $74 \%$ mortality $(n=14)$ and $21 \%$ mortality $(n=24)$ among the patients of ASA class IV and III respectively. Thirty-seven patients were operated on for emergency situations, of which 16 died (43\%) (Table 5). Such high mortality for emergency surgical procedures in patients with cirrhosis of liver has also been reported by Rice et al. ${ }^{1}$

\section{Limitations}

This study has certain limitations, including the use of a retrospective analysis approach and a small sample size. The number of patients who were denied surgery by the surgeon, anesthesiologist or the patients themselves based upon the expected high risk nature is not known and could have led to a selection bias towards surgical interventions in patients with less advanced or well-compensated liver disease.

\section{Conclusions}

High morbidity associated with surgical intervention in patients with cirrhosis of the liver warrants development of scientific models to prognosticate the outcome. The Mayo clinic risk calculator is good at predicting mortality but its efficacy in our patient population, we found, decreases with increase in duration after surgery. There is thus need to develop risk prognostication models suited for local populations. Until such time as these models are available, the Mayo risk calculation model may continue to be used with mortality risk cutoffs for the local population as derived in this study. MELD and MELD-Na scores and CTP classes may also be used

Table 5. Observed mortality and ASA status

\begin{tabular}{lllll}
\hline ASA class & Elective cases & Emergency cases & Mortality in emergency cases & Overall mortality \\
\hline III, $n=114$ & 92 & 22 & $4(19.3 \%)$ & $23(20.2 \%)$ \\
IV $n=19$ & 4 & 15 & $12(63.2 \%)$ & $14(73.7 \%)$ \\
\hline
\end{tabular}


Table 6. Type of non-transplant surgeries that patients with cirrhosis of the liver underwent

\begin{tabular}{ll}
\hline Type of surgery & Number of surgeries \\
\hline Gastrointestinal & 70 \\
Urological & 37 \\
Hepatic & 6 \\
Superficial & \\
I \& D & 10 \\
Trachestomy & 5 \\
ENT & 3 \\
Neuro & 2 \\
\hline
\end{tabular}

to prognosticate mortality risk in such patients with cirrhosis of the liver.

\section{Conflict of interest}

The authors have no conflict of interests related to this publication.

\section{Author contributions}

Conception and design of the study, assistance in analysis and review of the literature (MT), collection of data, review of the literature, and preparation of the first draft of the manuscript (KKKS), critical revision of the manuscript and provision of intellectual input (CKP), statistical analysis (PJ).

\section{References}

[1] Rice HE, O'Keefe GE, Helton WS, Johansen K. Morbid prognostic features in patients with chronic liver failure undergoing nonhepatic surgery. Arch Surg
1997;132:880-884; discussion 884-885. doi: 10.1001/archsurg.1997. 01430320082013.

[2] Friedman LS. The risk of surgery in patients with liver disease. Hepatology 1999;29:1617-1623. doi: 10.1002/hep.510290639.

[3] Patel T. Surgery in the patient with liver disease. Mayo Clin Proc 1999;74: 593-599. doi: 10.4065/74.6.593.

[4] Aranha GV, Sontag SJ, Greenlee HB. Cholecystectomy in cirrhotic patients: a formidable operation. Am J Surg 1982;143:55-60. doi: 10.1016/0002-9610 (82) $90129-5$.

[5] Keats AS. The ASA classification of physical status-a recapitulation. Anesthesiology 1978;49:233-236. doi: 10.1097/00000542-197810000-00001.

[6] Pugh RN, Murray-Lyon IM, Dawson JL, Pietroni MC, Williams R. Transection of the oesophagus for bleeding oesophageal varices. Br J Surg 1973;60:646649. doi: $10.1002 /$ bjs. 1800600817.

[7] Wiesner R, Edwards E, Freeman R, Harper A, Kim R, Kamath P, et al. Model for end-stage liver disease (MELD) and allocation of donor livers. Gastroenterology 2003;124:91-96. doi: 10.1053/gast.2003.50016.

[8] Kamath PS, Wiesner RH, Malinchoc M, Kremers W, Therneau TM, Kosberg CL, et al. A model to predict survival in patients with end-stage liver disease. Hepatology 2001;33:464-470. doi: 10.1053/jhep.2001.22172.

[9] Biggins SW, Kim WR, Terrault NA, Saab S, Balan V, Schiano T, et al. Evidencebased incorporation of serum sodium concentration into MELD. Gastroenterology 2006;130:1652-1660. doi: 10.1053/j.gastro.2006.02.010.

[10] Teh SH, Nagorney DM, Stevens SR, Offord KP, Therneau TM, Plevak DJ, et al. Risk factors for mortality after surgery in patients with cirrhosis. Gastroenterology 2007;132:1261-1269. doi: 10.1053/j.gastro.2007.01.040.

[11] Propst A, Propst T, Zangerl G, Ofner D, Judmaier G, Vogel W. Prognosis and life expectancy in chronic liver disease. Dig Dis Sci 1995;40:1805-1815. doi: 10.1007/bf02212706.

[12] Kim TH, Um SH, Yim SY, Seo YS, Yim HJ, Jeen YT, et al. The risk of perioperative adverse events in patients with chronic liver disease. Liver Int 2015; 35:713-723. doi: 10.1111/liv.12529.

[13] Garrison RN, Cryer HM, Howard DA, Polk HC Jr. Clarification of risk factors for abdominal operations in patients with hepatic cirrhosis. Ann Surg 1984;199: 648-655. doi: 10.1097/00000658-198406000-00003.

[14] Kim SY, Yim HJ, Park SM, Kim JH, Jung SW, Kim JH, et al. Validation of a Mayo post-operative mortality risk prediction model in Korean cirrhotic patients. Liver Int 2011;31:222-228. doi: 10.1111/j.1478-3231.2010.02419.x.

[15] Northup PG, Wanamaker RC, Lee VD, Adams RB, Berg CL. Model for EndStage Liver Disease (MELD) predicts nontransplant surgical mortality in patients with cirrhosis. Ann Surg 2005;242:244-251. doi: 10.1097/01.sla. 0000171327.29262.e0.

[16] Wehler M, Kokoska J, Reulbach U, Hahn EG, Strauss R. Short-term prognosis in critically ill patients with cirrhosis assessed by prognostic scoring systems. Hepatology 2001;34:255-261. doi: 10.1053/jhep.2001.26522. 